\title{
Joint Multiuser Detection and Optimal Spectrum Balancing for Digital Subscriber Lines
}

\author{
Vincent M. K. Chan and Wei Yu \\ The Edward S. Rogers Sr. Department of Electrical and Computer Engineering, \\ University of Toronto, Toronto, ON, Canada M5S $3 G 4$
}

Received 1 December 2004; Revised 27 April 2005; Accepted 8 July 2006

In a digital subscriber line (DSL) system with strong crosstalk, the detection and cancellation of interference signals have the potential to improve the overall data rate performance. However, as DSL crosstalk channels are highly frequency selective and multiuser detection is suitable only when crosstalk is strong, the set of frequency tones in which multiuser detection may be used must be carefully chosen. Further, this problem of tone selection is highly coupled with the transmit power spectra of both direct and interfering signals, so the optimal solution requires the tone selection problem to be solved jointly with the multiuser spectrum optimization problem. The main idea of this paper is that the above joint optimization may be done efficiently using a dual decomposition technique similar to that of the optimal spectrum balancing algorithm. Simulations show that multiuser detection can increase the bit rate performance in a remotely deployed ADSL environment. Rate improvement is also observed when near-end crosstalk is estimated and cancelled in a VDSL environment with overlapping upstream and downstream frequency bands.

Copyright @ 2006 Hindawi Publishing Corporation. All rights reserved.

\section{INTRODUCTION}

Crosstalk noise is a major limiting factor in wideband digital subscriber line (DSL) systems. Current research has focused on dynamic spectrum management (DSM) techniques for mitigating the effect of crosstalk [1]. The goal of DSM is to facilitate cooperation among mutually interfering lines in a binder. Cooperation may be implemented in two different levels. Power spectral density (PSD) level cooperation allows the optimal set of power spectral densities to be computed for each line in the binder so that the effect of mutual interference is minimized. In this case, multiple transmitters in a DSL binder operate independently, but at mutually accommodating PSD levels. The class of algorithms that are capable of computing the best set of PSDs is called spectrum balancing algorithms (e.g., $[2,3])$.

When cooperation is possible, not only at the PSD level, but also at the transmission signal level, the multiline DSL binder can then be truly designed as a multipleinput multiple-output (MIMO) system where multiuser detection algorithms can be implemented [4]. In this case, each line has the full knowledge of the transmitted signal from neighboring lines, and crosstalk can be completely cancelled. The capacity of a DSL binder with signal-level cooperation represents the ultimate capacity limit for DSL systems.

This paper explores a different form of cooperation that lies between the PSD-level and the signal-level cooperations described above. The algorithms described in this paper are most applicable to DSL configurations where the crosstalk channels are heavily unbalanced. For example, in a downstream ADSL deployment with an optical network unit (ONU), some remote terminals (RT) served from the central office (CO) can be located much closer to a nearby ONU than to their own CO. In this case, the crosstalk emitted by the ONU can overwhelm the intended transmission from the $\mathrm{CO}$. Hence, the crosstalk channel can be stronger than even the direct channel.

Signal-level cooperation is often not possible in the case described above. This is true for ADSL systems where the transmitters and the receivers are not physically colocated. In this case, PSD-level cooperation, although capable of producing a large gain as compared to the current practice of static spectrum management, is still not theoretically the best possible. The main point of this paper is that multiuser detection and crosstalk cancellation can bring further improvements to the system performance in these scenarios even when signal-level cooperation is not possible. 
One of the main contributions of this paper that enables crosstalk cancellation in systems with no signal-level cooperation is the idea of joint spectrum optimization and multiuser detection. Intuitively, crosstalk cancellation is effective only when the crosstalk signal is strong. In DSL systems, the crosstalk channels are usually more severe at high frequency tones. The crosstalk channel in the low frequency band is often too weak for crosstalk detection. Thus, multiuser detection must be carried out only at a selective set of tones for optimal performance. Further, the magnitude of crosstalk at each tone depends also on the transmit power spectra of the neighboring line at that tone. Hence, the problem of tone selection and the optimal multiuser spectrum balancing is strongly coupled. The main novelty of this paper is a method that determines the optimal transmit spectra jointly with the optimal tone selection for multiuser detection. The algorithm is based on the idea of dual optimization, recently applied to the optimal spectrum balancing problem in $[3,5]$ and its low-complexity version described in [6]. As the results of this paper show, multiuser detection can bring further improvement to the performance of the overall system beyond that of optimal spectral balancing alone without the need for additional cooperation.

The ideas of crosstalk cancellation and power allocation have been considered separately in the past. For example, [7] proposed a maximum-likelihood multiuser detector (ML-MUD) that considers all possible combinations of the interference signals and determines the most likely combination given the received signals. Alternatively, in an interference cancelling multiuser detector (IC-MUD), interference from adjacent users can be estimated, reconstructed, and subtracted from the received signal. It is shown in [8] that this type of interference cancelling scheme can achieve a substantial performance gain for near-end crosstalk cancellation. In terms of power allocation, [9] proposed an efficient method for allocating power in DSL systems with multiuser detection. However, crosstalk is assumed to be strong and crosstalk cancellation is performed in all channels. Hence, none of the previous work considers the joint optimization of bit/power allocation and crosstalk cancellation. The main contribution of this paper is to show that such a joint optimization can be done in a numerically efficient way.

While the crosstalk cancellation schemes mentioned in the above paragraphs involves full detection of the interference signal, this paper explores the possibility of performing partial detection as well. The idea of partial detection stems from classical information theoretical treatment of interference channel capacity. The largest achievable rate region for a Gaussian interference channel is described in $[10,11]$. The main idea of $[10,11]$ is that the detection and subtraction of the interfering signal is useful and that partial detection can further expand the rate region offered by complete detection. However, information theoretical results deal with frequency-flat channels only. This paper investigates the best achievable rate region for frequency-selective channels where the optimal power allocation across the frequency is of crucial importance.
The following assumptions are made in the rest of the paper. Perfect knowledge of channel state information of the direct and crosstalk channels is assumed. PSDlevel coordination between $\mathrm{CO}$ and $\mathrm{ONU}$ is assumed to be available for computing the best set of power spectra. The multiuser detection scheme used in the algorithm is of the interference cancelling type, in which the interfering signals are either detected fully or partially. Implementing this type of detection requires the assumption that the multiuser detector can perfectly synchronize with the interfering users, for example, using schemes described in $[12,13]$. Discrete multitone modulation (DMT) is assumed. Proper insertion of the cyclic prefix and suffix is assumed to ensure orthogonality between the DMT subchannels.

\section{OPTIMAL SPECTRUM BALANCING ALGORITHMS}

Before addressing the multiuser detection problem, it is useful to review the spectrum optimization problem without multiuser detection and to outline an existing algorithm called optimal spectrum balancing (OSB). The OSB algorithm solves the spectrum optimization problem in a computationally manageable fashion. It is a crucial ingredient for the joint multiuser detection and spectrum balancing algorithm to be described later.

\subsection{The spectrum optimization problem}

In a $K$-user DSL bundle, the objective of spectrum optimization is to maximize the weighted sum-rate of all participating users given an individual power constraint for each user. Given $\mathbf{P}_{\mathbf{k}}$ the power constraints for user $k$ and a set of weights $w_{k}$ such that $\sum_{k=1}^{K} w_{k}=1$, the goal of optimization is to find the set of $S_{k}^{n}$, which is the subchannel power for user $k$ in tone $n$, that maximizes the weighted sum of transmission rates of all users. Mathematically, the problem can be written as follows:

$$
\max _{\left\{S_{1}^{n}, \ldots, S_{K}^{n}\right\}_{n=1}^{N}} \sum_{k=1}^{K} w_{k} R_{k} \text { s.t. } P_{k} \leq \mathbf{P}_{\mathbf{k}} \quad \forall k,
$$

where $P_{k}$ is the total power used by user $k, R_{k}$ is the total rate achieved by user $k$, and $N$ is the number of frequency tones in the DMT system. Solving (1) for all combinations of $w_{k}$ gives the achievable rate region of the system. The design variables in this problem are $S_{k}^{n}$ s subject to the constraints

$$
P_{k}=\Delta f \sum_{n=1}^{N} S_{k}^{n} \leq \mathbf{P}_{\mathbf{k}}
$$

and $S_{k}^{n} \geq 0$, for all $k, n$ where $\Delta f$ is the frequency width of the DMT tones. Since DMT modulation facilitates independent data transmission on each tone, $R_{k}$ in (1) can be calculated 
as $R_{k}=(1 / T) \sum_{n=1}^{N} b_{k}^{n}$, where $T$ is the symbol period and $b_{k}^{n}$ denotes the achievable bit rate for user $k$ in tone $n$ given by

$$
b_{k}^{n}=\left\lfloor\log _{2}\left(1+\frac{1}{\Gamma} \cdot \frac{\left|h_{k}^{n}\right|^{2} S_{k}^{n}}{\sigma_{k}^{n}+\sum_{i \neq k}\left|\alpha_{i, k}^{n}\right|^{2} S_{i}^{n}}\right)\right\rfloor .
$$

Here, $\Gamma$ is the SNR gap, $\sigma_{k}^{n}$ is the channel noise variance for user $k$ in tone $n, h_{k}^{n}$ is the direct channel transfer function for user $k$ in tone $n$, and $\alpha_{i, k}^{n}$ is the crosstalk transfer function from the $i$ th user to the $k$ th user in tone $n$.

The following assumptions are made in the above rate calculation. First, discrete bit-loading is assumed, meaning that the number of bits loaded into each tone is restricted to be integer values. Second, a transmitted signal from one user is always treated as noise for all other users. The possibility of crosstalk cancellation and multiuser detection is disregarded. Third, intertone interference caused by channel propagation delay and unsynchronous DMT blocks is neglected. This assumption is reasonable as long as the intertone interference is minimized in practical frame-synchronous systems implementing zipper-like modulation $[12,13]$. With the last two assumptions, the signal received by user $k$ contains crosstalk interference from all other users on a tone-by-tone basis.

\subsection{Optimal spectrum balancing}

The main difficulty of the spectrum optimization problem (1) is that $R_{k}$ is a nonconvex function of $S_{k}^{n}$. As the optimization is coupled over frequency by the power constraints, solving this problem with a brute-force approach involves searching through all possible bit allocations on all frequency tones. This requires a complexity that is exponential in $N$, where $N=256$ for ADSL and $N=4096$ for VDSL systems. Clearly, this is computationally intractable in a practical implementation.

To reduce the computational complexity, the OSB algorithm proposed in [3] uses the idea of dual decomposition and solves the problem in the Lagrangian dual-domain. The main idea is to form the dual of the original problem and to decompose the dual problem on a tone-by-tone basis. The dual problem is the optimization of $\min _{\lambda_{1}, \ldots, \lambda_{K}} g\left(\lambda_{1}, \ldots, \lambda_{K}\right)$ subject to $\lambda_{k} \geq 0$. Hence, solving the dual problem consists of evaluating the dual objective $g\left(\lambda_{1}, \ldots, \lambda_{K}\right)$ for fixed $\left\{\lambda_{1}, \ldots, \lambda_{K}\right\}$ and minimizing $g\left(\lambda_{1}, \ldots, \lambda_{K}\right)$ over nonnegative $\lambda_{k}$ 's.

The evaluation of $g\left(\lambda_{1}, \ldots, \lambda_{K}\right)$ can be simplified by decomposing the dual objective as follows:

$$
\begin{aligned}
& g\left(\lambda_{1}, \ldots, \lambda_{K}\right) \\
& =\max _{\left\{S_{1}^{n}, \ldots, S_{K}^{n}\right\}_{n=1}^{N}} \sum_{k=1}^{K} w_{k} R_{k}-\sum_{k=1}^{K} \lambda_{k}\left(P_{k}-\mathbf{P}_{\mathbf{k}}\right) \\
& \quad=\left(\sum_{n=1}^{N} \max _{S_{1}^{n}, \ldots, S_{K}^{n}} \sum_{k=1}^{K}\left(w_{k} b_{k}^{n}-\lambda_{k} S_{k}^{n}\right)\right)+\sum_{k=1}^{K} \lambda_{k} \mathbf{P}_{\mathbf{k}} .
\end{aligned}
$$

It turns out that for the spectrum optimization problem in DMT systems, the duality gap is zero even though the primal problem is nonconvex [5]. The reason is that all DMT-based systems satisfy a so-called time-sharing property which essentially transforms the nonconvex objective function into a convex function. More precisely, given the total power of two power allocation schemes $P_{x}, P_{y}$, let $R(P)$ denote the maximum rate achievable using $P$. The requirement of the time-sharing property is that all intermediate rate $v R\left(P_{x}\right)+(1-v) R\left(P_{y}\right)$ must be achievable using $v P_{x}+(1-v) P_{y}$ (where $0 \leq v \leq 1$ is the timesharing variable). The time-sharing property ensures that $R(P)$ is concave in $P$, which in turn ensures the zero duality gap.

The DMT systems satisfy the time-sharing property whenever the frequency tone spacing is small. In this case, the intermediate rate can be achieved by interleaving the frequency tones of the two original power allocations corresponding to $R\left(P_{x}\right)$ and $R\left(P_{y}\right)$. The approximation is accurate as long as $N$ is sufficiently large, which is true for practical DSL systems. 


\subsection{Iterative spectrum balancing}

Although the complexity of OSB is linear in $N$, the optimization within each tone, namely $\max _{S_{1}^{n}, \ldots, S_{K}^{n}} \sum_{k=1}^{K}\left(w_{k} b_{k}^{n}-\lambda_{k} S_{k}^{n}\right)$, has exponential complexity in $K$. To further reduce this complexity, an approximate near-optimal iterative spectrum balancing (ISB) algorithm is devised in [6]. The main idea of the ISB is to evaluate (4) approximately by iteratively optimizing $\sum_{k=1}^{K}\left(w_{k} b_{k}^{n}-\lambda_{k} S_{k}^{n}\right)$ on a user-by-user basis. Specifically, the following maximization is performed repeatedly until convergence:

$$
\max _{S_{K}^{n}} \cdots \max _{S_{2}^{n}} \max _{S_{1}^{n}} \sum_{k=1}^{K}\left(w_{k} b_{k}^{n}-\lambda_{k} S_{k}^{n}\right) .
$$

Hence, the algorithm first optimizes $S_{1}^{n}$ while keeping $S_{2}^{n}, \ldots, S_{K}^{n}$ fixed, then optimizes $S_{2}^{n}$ keeping all other $S_{k}^{n}$ fixed, then $S_{3}^{n}, \ldots, S_{K}^{n}$, then $S_{1}^{n}, S_{2}^{n}, \ldots$, and so on. Convergence is guaranteed because the objective function is nondecreasing in each iteration. Although not globally optimal, simulation shows that this scheme provides a near-optimal performance as compared to OSB for many practical channels.

The major advantage that ISB offers over OSB is that its computational complexity is polynomial in the number of users (and linear in the number of tones as before). OSB is not practical when the number of users is large. However, ISB can be applied to a large number of users while providing a substantial performance gain to that of conventional methods such as iterative water-filling [2].

\section{JOINT MULTIUSER DETECTION AND OPTIMAL SPECTRUM BALANCING}

In both spectrum balancing algorithms, as described in the previous section, crosstalk from adjacent users is always regarded as noise. This is near-optimal when the crosstalk channel gains, $\alpha_{i, k}^{n}$ for $i \neq k$, are small. In many practical circumstances, however, an interfering transmitter can be located very closely to the receiver of a neighboring user, for example, see Figure 1. In this case, crosstalk cancellation schemes as described in the following sections may potentially bring additional performance gains. The discussion in this section is restricted to the detection of far-end crosstalks (FEXT). Near-end crosstalk (NEXT) cancellation will be addressed later.

\subsection{Full detection of the interfering user}

The main idea proposed in this paper is that multiuser detectors (MUD) can be applied in conjunction with spectrum optimization in situations such as that in Figure 1. A multiuser detector at the receiver of user 1 works by first detecting and subtracting the signal from user 2 in the received signal, then detecting the signal from user 1. Implementation of this scheme requires error-free decoding of user 2 at user 1 .

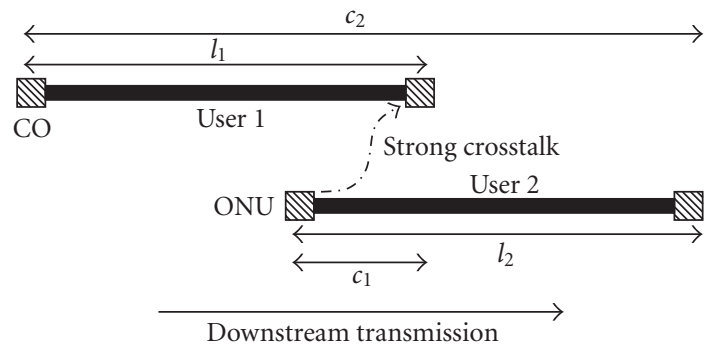

FIGURE 1: Loop topology for 2-user ADSL downstream.

Thus, the bit rate of user 2 is restricted by the quality of the crosstalk channel. Therefore,

$$
\begin{aligned}
\tilde{b}_{1}^{n}= & \left\lfloor\log _{2}\left(1+\frac{1}{\Gamma} \cdot \frac{\left|h_{1}^{n}\right|^{2} S_{1}^{n}}{\sigma_{1}^{n}}\right)\right\rfloor \\
\tilde{b}_{2}^{n}=\min ( & \left\lfloor\log _{2}\left(1+\frac{1}{\Gamma} \cdot \frac{\left|\alpha_{2,1}^{n}\right|^{2} S_{2}^{n}}{\sigma_{1}^{n}+\left|h_{1}^{n}\right|^{2} S_{1}^{n}}\right)\right\rfloor, \\
& \left.\left\lfloor\log _{2}\left(1+\frac{1}{\Gamma} \cdot \frac{\left|h_{2}^{n}\right|^{2} S_{2}^{n}}{\sigma_{2}^{n}+\left|\alpha_{1,2}^{n}\right|^{2} S_{1}^{n}}\right)\right\rfloor\right)
\end{aligned}
$$

is an achievable rate pair. Note the removal of the $\left|\alpha_{2,1}^{n}\right|^{2} S_{2}^{n}$ term in the noise of $\tilde{b}_{1}^{n}$ due to crosstalk cancellation. Thus, $\tilde{b}_{1}^{n}$ is now larger than before. However, to ensure that $\tilde{b}_{2}^{n}$ may be cancelled by the first user, $\tilde{b}_{2}^{n}$ is now the minimum of the rate allowed by the crosstalk channel $\left\lfloor\log _{2}(1+(1 / \Gamma)\right.$. $\left.\left.\left(\left|\alpha_{2,1}^{n}\right|^{2} S_{2}^{n} /\left(\sigma_{1}^{n}+\left|h_{1}^{n}\right|^{2} S_{1}^{n}\right)\right)\right)\right\rfloor$ and the rate of the direct channel $\left\lfloor\log _{2}\left(1+(1 / \Gamma) \cdot\left(\left|h_{2}^{n}\right|^{2} S_{2}^{n} /\left(\sigma_{2}^{n}+\left|\alpha_{1,2}^{n}\right|^{2} S_{1}^{n}\right)\right)\right)\right\rfloor$.

Since channel gains are frequency selective, not every tone in the crosstalk channel is suitable for multiuser detection. Good quality crosstalk channels, or channels with large $\alpha_{2,1}^{n}$, only reside in the high frequencies where the crosstalk coupling between lines is strong. Thus, the multiuser detection scheme is effective when it is applied only to high frequency tones. Making such a tone selection for multiuser detection is not trivial but important for achieving the optimal weighted sum-rate.

This paper proposes a method that jointly determines the optimal tone selection and optimal spectrum in an efficient manner. The method is based on the dual decomposition idea of the OSB algorithm. For any tone $n$, multiuser detection at receiver 1 can be enabled or disabled. This provides an alternative mapping function from $\left\{S_{1}^{n}, \ldots, S_{K}^{n}\right\}$ to $\left\{b_{1}^{n}, \ldots, b_{K}^{n}\right\}$. The choice between the two for each tone is the one that maximizes $g\left(\lambda_{1}, \ldots, \lambda_{K}\right)$. When $K=2$, (4) can be modified as follows:

$$
\begin{aligned}
g\left(\lambda_{1}, \lambda_{2}\right) & \\
= & \left(\sum_{n=1}^{N} \max _{S_{1}^{n}, S_{2}^{n}}\left(\max \left(\sum_{k=1}^{2} w_{k} b_{k}^{n}, \sum_{k=1}^{2} w_{k} \tilde{b}_{k}^{n}\right)-\sum_{k=1}^{2} \lambda_{k} S_{k}^{n}\right)\right) \\
& +\sum_{k=1}^{2} \lambda_{k} \mathbf{P}_{\mathbf{k}} .
\end{aligned}
$$




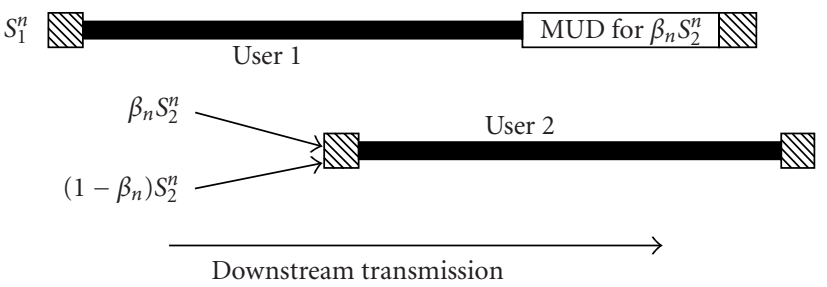

Figure 2: Partial interference detection for 2-user ADSL downstream.

The set $\left\{S_{1}^{n}, S_{2}^{n}\right\}$ that minimizes $g\left(\lambda_{1}, \lambda_{2}\right)$ is the optimal power spectra and the choice of $b_{k}^{n}$ or $b_{k}^{n}$ in the inner maximization determines the MUD mode for tone $n$. Similar to optimal spectrum balancing, the search for optimal $\left\{S_{1}^{n}, S_{2}^{n}\right\}$ can be performed by searching for the optimal $\left\{b_{1}^{n}, b_{2}^{n}\right\}$ or $\left\{\tilde{b}_{1}^{n}, \tilde{b}_{2}^{n}\right\}$ and inverting (7) to obtain the corresponding $\left\{S_{1}^{n}, S_{2}^{n}\right\}$. Although an extra maximization computation is required when multiuser detection is taken into account, the order of complexity remains at $O\left(N B^{2}\right)$.

Same as in the case of OSB, The joint multiuser detection and optimal spectrum balancing algorithm works by minimizing $g\left(\lambda_{1}, \lambda_{2}\right)$ over all $\lambda_{k}$ 's using a subgradient algorithm. When $N \rightarrow \infty$, in which case the time-sharing property of the DMT system holds, global optimality of this algorithm is guaranteed, as shown in the following theorem.

Theorem 1. The joint multiuser detection and optimal spectrum balancing algorithm achieves global optimality in the spectrum optimization problem (1) as $N \rightarrow \infty$.

Proof. The frequency tone spacing approaches zero as $N \rightarrow$ $\infty$. In this case, the DMT system can achieve the time-sharing property by using the frequency tone interleaving scheme described in Section 2.2. This reduces the duality gap to zero. Hence, global optimality can be achieved by minimizing the dual objective $g\left(\lambda_{1}, \lambda_{2}\right)$. Since the dual objective is always convex, global optimum can always be reached by using a subgradient search.

This proof of global optimality is similar to that of the OSB algorithm. The inclusion of the alternative mapping $\left\{\tilde{b}_{k}^{n}\right\}$ does not affect the convexity of the primal objective with respect to the power constraint. As long as $g\left(\lambda_{1}, \lambda_{2}\right)$ is evaluated by maximizing over all $\left\{b_{1}^{n}, b_{2}^{n}\right\}$ and $\left\{\tilde{b}_{1}^{n}, \tilde{b}_{2}^{n}\right\}$, global optimum can be reached by minimizing $g\left(\lambda_{1}, \lambda_{2}\right)$.

For a general 2-user interference channel, an MUD can be installed at both/either/neither receivers, resulting in a total of four options. However, the placement of MUD can often be easily determined for practical channels given the channel lengths. Referring to Figure 1, simulation experience shows that an MUD at user $i$ is effective only if $c_{i} / l_{i}<1$. Clearly, it is not possible that both $c_{1}<l_{1}$ and $c_{2}<l_{2}$. Hence, the possibility of using two MUDs can be eliminated, and the MUD should only be placed at user $i$ with a smaller $c_{i} / l_{i}$. The decision of whether an MUD should be used at all depends on the extra receiver complexity required and the performance gain obtained. The simulations in the later section illustrate the benefit of multiuser detection as a function of the length of the crosstalk channel.
The above method for finding the optimal power spectrum with MUD at the receivers can be extended to more than two users. However, the algorithm does become more complex. With two users, as in previous example, there are only two modes for the MUD: either cancelling or ignoring the crosstalk. If instead there are $S$ users connecting to CO and $T$ users connecting to ONU in Figure 1, there are $S T$ cancellable strong crosstalk channels, giving a total of $2^{S T}$ MUD modes. To lower the complexity, an upper limit should be imposed on the number of crosstalk channels considered for cancellation while the rest of the crosstalk channels should be ignored for cancellation. Choosing which crosstalk should be ignored depends on the actual channel configurations. Nevertheless, once the choice of cancellable crosstalk is made offline, the joint multiuser detection and OSB algorithm determines the optimal spectra efficiently.

So far, the type of multiuser detection described involves fully resolving the signals transmitted from the interfering user. Intuitively, this imposes a strict upper bound on the bit rate of user 2. To relax this restriction, a scheme that involves only partial detection of the interfering user is introduced in the next section.

\subsection{Partial detection of the interfering user}

In a 2-user interference channel, partial detection of the signal from user 2 at user 1 on tone $n$ works by first partitioning the bitstream at transmitter 2 and then allocating $\beta_{n} S_{k}^{n}$ and $\left(1-\beta_{n}\right) S_{k}^{n}$ to the two streams. Here, $\beta_{n}$ denotes the fraction of signal power at user 2 intended for multiuser detection. The two bitstreams are modulated separately and transmitted through the same channel, as illustrated in Figure 2.

One possible scheme for implementing bitstream partitioning is nested signal constellation. Suppose $b_{2, \beta}^{n}$ are the bits resulting from $\beta_{n} S_{k}^{n}$, which are designed for multiuser detection by user 1 , and $b_{2, \bar{\beta}}^{n}$ are the undetected bits resulting from $\left(1-\beta_{n}\right) S_{k}^{n}$. The $b_{2, \beta}^{n}$ bits are first modulated in a $2^{b_{2, \beta}^{n}}$ points constellation. Each signal point is yet another constellation with $2^{b_{2, \bar{\beta}}^{n}}$ signal points. Then, user 1 only tries to detect $b_{2, \beta}^{n}$ bits while seeing the other $b_{2, \bar{\beta}}^{n}$ bits as noise; user 2 treats the nested constellation as a single constellation and performs the full detection of $b_{2, \beta}^{n}+b_{2, \bar{\beta}}^{n}$ bits. This scheme requires the restriction that both $b_{2, \beta}^{n}$ and $b_{2, \bar{\beta}}^{n}$ are of integer values.

When the option of partial detection is enabled, (3) and (7) can be modified to 


$$
\begin{aligned}
\tilde{b}_{1}^{n}\left(\beta_{n}\right)= & \left\lfloor\log _{2}\left(1+\frac{1}{\Gamma} \cdot \frac{\left|h_{1}^{n}\right|^{2} S_{1}^{n}}{\sigma_{1}^{n}+\left|\alpha_{2,1}^{n}\right|^{2}\left(1-\beta_{n}\right) S_{2}^{n}}\right)\right\rfloor \\
\tilde{b}_{2}^{n}\left(\beta_{n}\right)= & \left\lfloor\log _{2}\left(1+\frac{1}{\Gamma} \cdot \frac{\left|h_{2}^{n}\right|^{2}\left(1-\beta_{n}\right) S_{2}^{n}}{\sigma_{2}^{n}+\left|\alpha_{1,2}^{n}\right|^{2} S_{1}^{n}}\right)\right\rfloor+\min \left(\left\lfloor\log _{2}\left(1+\frac{1}{\Gamma} \cdot \frac{\left|\alpha_{2,1}^{n}\right|^{2} \beta_{n} S_{2}^{n}}{\sigma_{1}^{n}+\left|h_{1}^{n}\right|^{2} S_{1}^{n}+\left|\alpha_{2,1}^{n}\right|^{2}\left(1-\beta_{n}\right) S_{2}^{n}}\right)\right\rfloor,\right. \\
& \left.\left\lfloor\log _{2}\left(1+\frac{1}{\Gamma} \cdot \frac{\left|h_{2}^{n}\right|^{2} \beta_{n} S_{2}^{n}}{\sigma_{2}^{n}+\left|\alpha_{1,2}^{n}\right|^{2} S_{1}^{n}+\left|h_{2}^{n}\right|^{2}\left(1-\beta_{n}\right) S_{2}^{n}}\right)\right\rfloor\right) .
\end{aligned}
$$

In (9), $\beta_{n}$ represents a continuum between no multiuser detection at user 1 and full detection of user 2. When $\beta_{n}=0$, (9) can be reduced to (3). Similarly, when $\beta_{n}=1$, (9) can be reduced to (7).

Similar to the case of full detection, incorporating (9) into the OSB algorithm requires solving $N$ per-tone maximization of the dual objective over $\left\{S_{1}^{n}, \ldots, S_{K}^{n}\right\}$ and $\beta^{n}$. The dual objective for a 2-user system becomes

$$
\begin{aligned}
& g\left(\lambda_{1}, \lambda_{2}\right) \\
& =\left(\sum_{n=1}^{N} \max _{S_{1}^{n}, S_{2}^{n}, \beta_{n}}\left(\sum_{k=1}^{2} w_{k} b_{k}^{n}\left(\beta_{n}\right)-\sum_{k=1}^{2} \lambda_{k} S_{k}^{n}\right)\right) \\
& +\sum_{k=1}^{2} \lambda_{k} \mathbf{P}_{\mathbf{k}} .
\end{aligned}
$$

An exhaustive search over $\left\{S_{1}^{n}, S_{2}^{n}, \beta_{n}\right\}$ is feasible because we only allow integer bitstream partitioning at user 2 . Then, the search space of $\left\{S_{1}^{n}, S_{2}^{n}, \beta_{n}\right\}$ is equivalent to that of $\left\{b_{1}^{n}, b_{2, \bar{\beta}}^{n}, b_{2, \beta}^{n}\right\}$. The complexity of the this scheme for 2 -user systems becomes $O\left(N B^{3}\right)$.

Since the optimization space includes cases of $\beta_{n}=0$ and $\beta_{n}=1$, this partial detection scheme performs at least as well as full detection. However, simulation results show that the option of partial detection only provides marginal performance gain for DSL systems. Given the increase in transceiver complexity involved, allowing partial detection is not necessary for DSL systems.

\section{JOINT MULTIUSER DETECTION AND ITERATIVE SPECTRUM BALANCING}

The complexity of evaluating $g\left(\lambda_{1}, \ldots, \lambda_{K}\right)$ in the optimal spectrum balancing algorithm may be reduced by applying ISB, the iterative (and near-optimal) approach. A similar approach can be applied when multiuser detection is considered. The following section describes a scheme that works with a 2-user system operating downstream transmission as in Figure 1 when only full detection is considered.

The algorithm involves evaluating $g\left(\lambda_{1}, \lambda_{2}\right)$ from (8) in an iterative fashion. For a fixed set of $\lambda_{k}$ 's, $g\left(\lambda_{1}, \lambda_{2}\right)$ is maximized over $S_{1}^{n}$ while holding $S_{2}^{n}$ constant. Then the maximization is performed over $S_{2}^{n}$, and this continues between $S_{1}^{n}$ and $S_{2}^{n}$ until it converges. This means that the following pertone maximization problems will be carried out alternately:

$$
\begin{aligned}
& \max _{S_{1}^{n}}\left(\max \left(\sum_{k=1}^{2} w_{k} b_{k}^{n}, \sum_{k=1}^{2} w_{k} \tilde{b}_{k}^{n}\right)\right)-\lambda_{1} S_{1}^{n}, \\
& \max _{S_{2}^{n}}\left(\max \left(\sum_{k=1}^{2} w_{k} b_{k}^{n}, \sum_{k=1}^{2} w_{k} \tilde{b}_{k}^{n}\right)\right)-\lambda_{2} S_{2}^{n} .
\end{aligned}
$$

Same as in the case of ISB, this iterative algorithm always converges because $g\left(\lambda_{1}, \lambda_{2}\right)$ is nondecreasing for each iteration. In terms of implementation, the maximization over $S_{k}^{n}$ can be once again performed by maximizing over $b_{k}^{n}$. Although this iterative technique cannot retain the linear complexity of ISB due to exponentially growing number of MUD modes, this technique has drastically reduced the complexity from that of the joint multiuser detection and OSB algorithm.

The idea of running ISB with multiuser detection can be extended to systems with more than 2 users. However, the multiuser detection scheme becomes much more complex when $K$ is large. In general, there are $2\left(\begin{array}{c}K \\ 2\end{array}\right)$ crosstalk channels in a $K$-user frequency-division duplex system. Although only the strong crosstalk requires participation in the multiuser detection scheme, the number of MUD modes still increases drastically with $K$. Hence, the number of crosstalk channels considered for cancellation must be limited for complexity concerns.

\section{NEAR-END CROSSTALK CANCELLATION IN FULL DUPLEX DSL SYSTEMS}

In traditional DSL system design, upstream and downstream transmissions are usually separated with a frequencydivision duplex scheme in order to avoid near-end crosstalk. With multiuser detection, near-end crosstalk can potentially be detected and cancelled. This gives rise to the possibility of a fully duplex DSL system.

Consider a 2-user VDSL system as shown in Figure 3 in which both upstream and downstream transmission takes place simultaneously in the same frequency band. There are a total of four transmitters. The joint spectrum balancing and multiuser detection algorithm described in the previous 


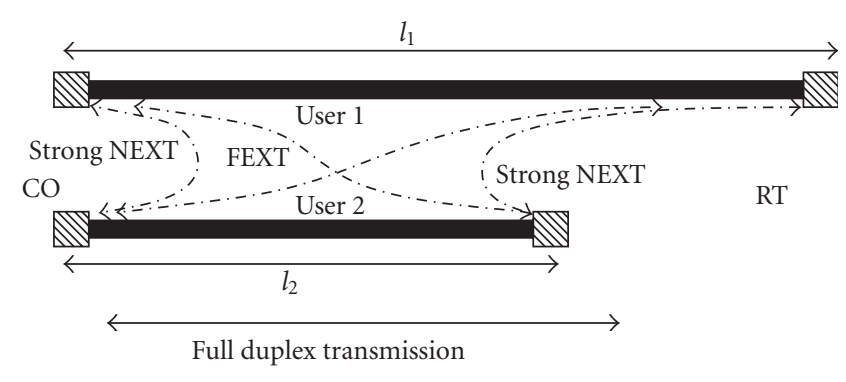

Figure 3: Loop topology for 2-user full duplex VDSL.

section can be directly applied to this case by considering an equivalent 4-user system with 8 crosstalk channels.

Let $S_{1}^{n}$ and $S_{2}^{n}$ be the downstream transmission powers for users 1 and 2, respectively. Let $S_{3}^{n}, S_{4}^{n}$ be the upstream transmission power for users 1 and 2. Let the FEXT channels be $\alpha_{1,2}^{n}, \alpha_{2,1}^{n}, \alpha_{3,4}^{n}, \alpha_{4,3}^{n}$, and the NEXT channels be $\alpha_{1,4}^{n}, \alpha_{4,1}^{n}, \alpha_{2,3}^{n}$, $\alpha_{3,2}^{n}$. Assume perfect echo cancellation. So, the rest of the $\alpha_{i, k}^{n}$ 's are also zero. The equivalent 4 -user system has 8 crosstalk channels, and thus $2^{8}$ MUD modes. However, the rate equation for a particular user is primarily affected by only 2 of the crosstalk channels, one of them being NEXT and the other being FEXT. For example, the bit rate $b_{1}^{n}$ derived from $S_{1}^{n}$ is only affected by FEXT from $\alpha_{2,1}^{n}$ and NEXT from $\alpha_{4,1}^{n}$. In addition, the assumption that FEXT is much smaller than NEXT in the configuration of Figure 3 can be safely taken. Hence, crosstalk cancellation from only one NEXT channel should be considered.

Simulation results in the next section show rate improvement when NEXT cancellation is performed in a 2-user VDSL full duplex system. This suggests potential grounds for improvement of the current VDSL system with a fixed nonoverlapping bandplan for upstream and downstream.

\section{SIMULATIONS}

This section illustrates the improvement in bit rate with multiuser detection. The performances of the joint optimal spectrum balancing and the joint iterative spectrum balancing algorithms are simulated in DSL binders. For all simulations except where specified, a target error probability of $10^{-7}$ with about $3 \mathrm{~dB}$ coding gain and $6 \mathrm{~dB}$ noise margin is used. The DSL lines are 26-gauge twisted pairs for all cases.

\subsection{ADSL downstream}

A 2-user ADSL downstream scenario as shown in Figure 1 with $l_{1}=l_{2}=12 \mathrm{kft}$ and $c_{1}=1 \mathrm{kft}$ is simulated. The crosstalk from transmitter 2 to receiver 1 is large due to the close distance between them. The FEXT channel is simulated using standard methods. It represents the $99 \%$ worstcase crosstalk scenario. Figure 4 shows the strength of the direct and crosstalk channels. As clearly illustrated in the figure, crosstalk is weak at low frequency but it overwhelms the

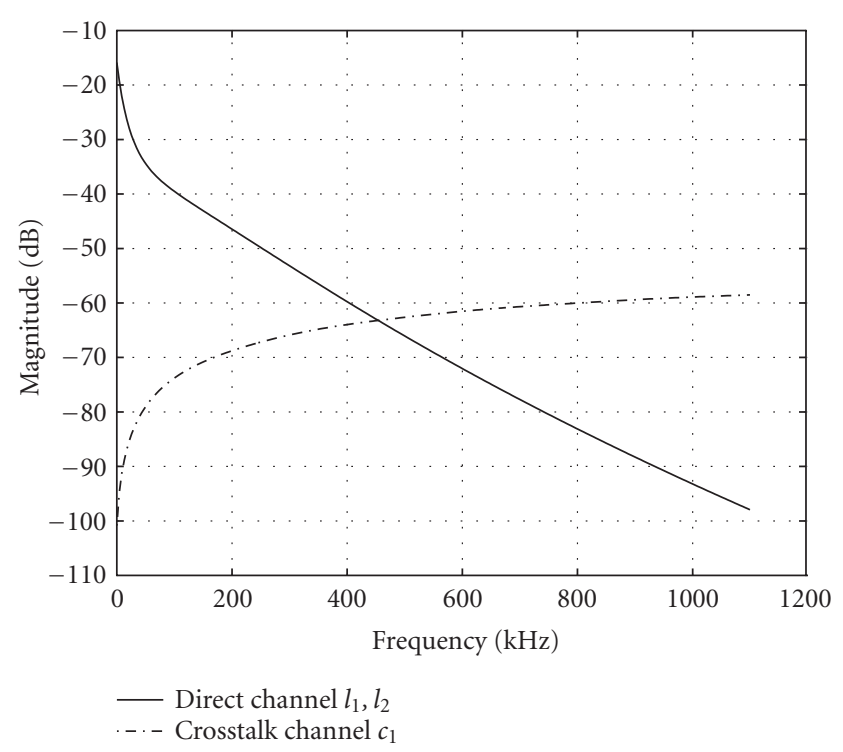

Figure 4: Channel response of $12 \mathrm{kft}$ direct channels and $1 \mathrm{kft}$ crosstalk channel.

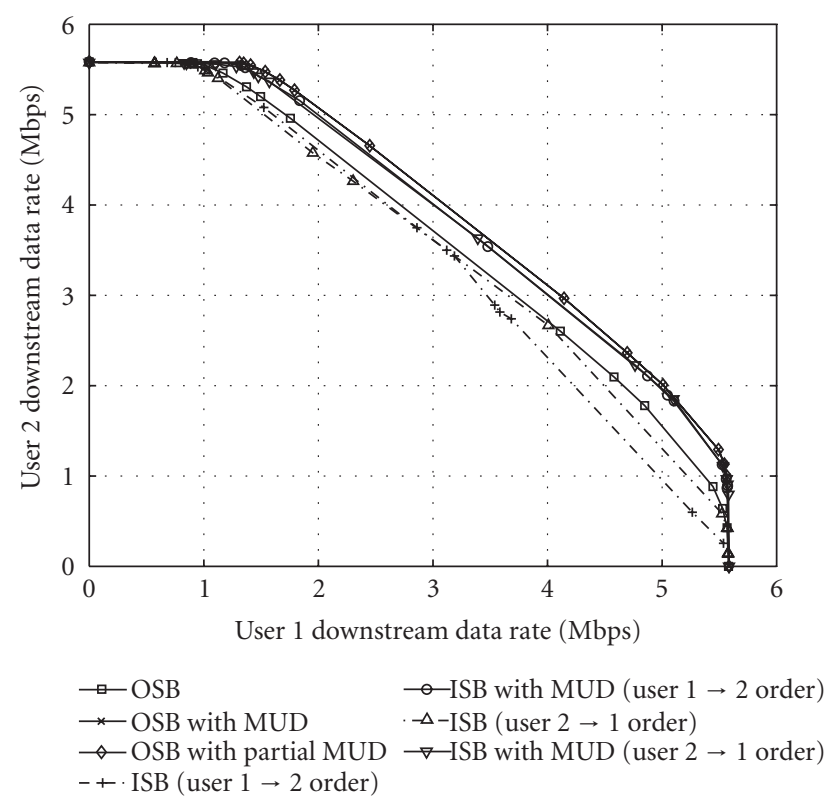

FIgURE 5: Achievable rate region for 2-user ADSL downstream using OSB/ISB and the joint multiuser detection algorithm for gap = $12 \mathrm{~dB}$.

direct channel at high frequency. Thus, multiuser detection should only be performed at high frequency tones. Note that the ideal tone selection for crosstalk cancellation depends on not only the channel response, but also the transmission power of the interfering user. The joint multiuser detection algorithms proposed in this paper solve the coupling problem of tone selection and power allocation simultaneously in an efficient manner.

Figure 5 shows the achievable rate increase offered by the joint multiuser detection algorithm. When OSB is performed 


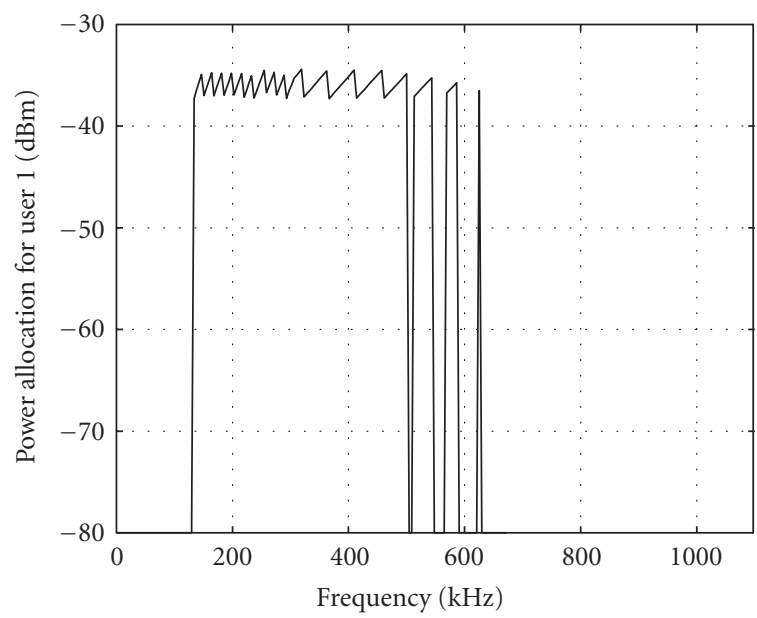

(a)

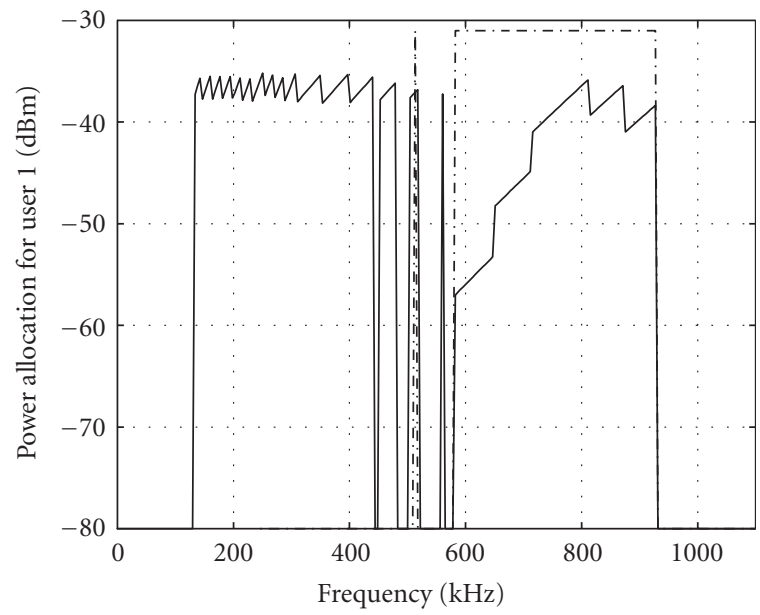

(c)

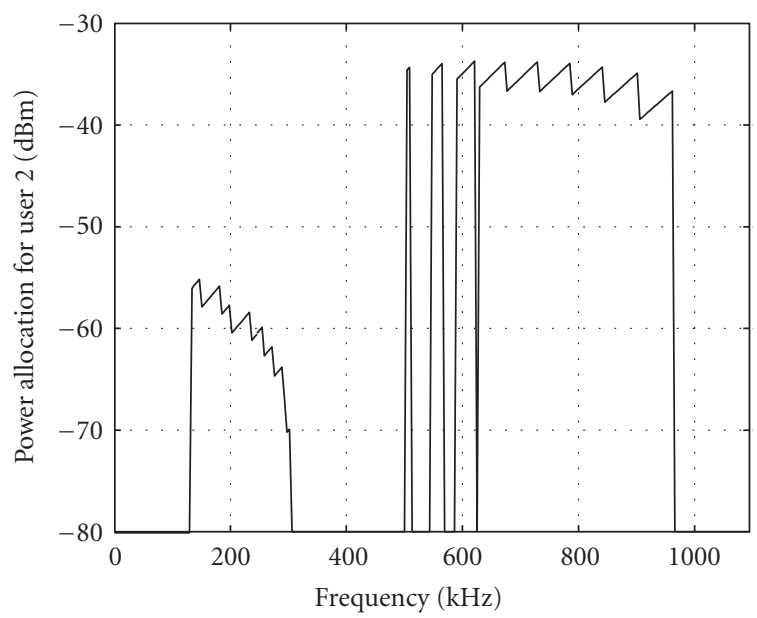

(b)

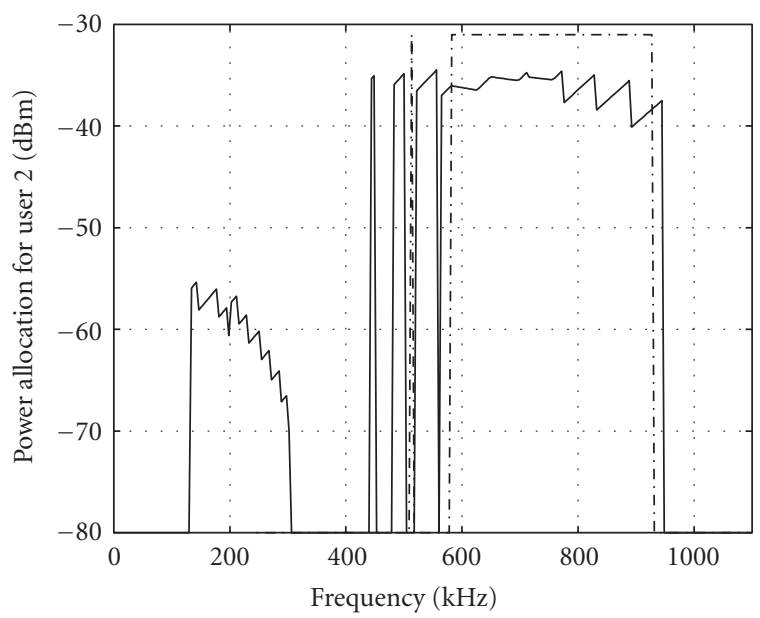

(d)

FIgURE 6: Power allocations for 2-user ADSL downstream (a) and (b) with optimal spectrum balancing alone and (c) and (d) with the joint multiuser detection algorithm (a) and (b) correspond to the rate pair $R_{1}=4.1120 \mathrm{Mbps}, R_{2}=2.6040 \mathrm{Mbps}$; and (c) and (d) correspond to the rate pair $R_{1}=4.1440 \mathrm{Mbps}, R_{2}=2.9680 \mathrm{Mbps}$. The dotted line denotes the frequency band in which multiuser detection is applied. Full interference detection is assumed.

with multiuser detection, a $14 \%$ increase for one user or $7 \%$ for both users can be observed. For example, without multiuser detection (4.1120 Mbps, 2.6040 Mbps) is achievable; with multiuser detection it is increased to $(4.1440 \mathrm{Mbps}$, $2.9680 \mathrm{Mbps}$ ). The corresponding power allocation for both users at these rates are illustrated in Figure 6. Note that in high frequency bands, frequency-division multiplexing for the two users is enforced when MUD is off. On the other hand, joint multiuser detection and spectrum balancing allows both users to transmit data even when crosstalk is severe at high frequency. The extra bits transmitted in this region contribute to the overall bit rate increase.
The following further observations can be made. As mentioned in previous sections, the rate region offered by partial detection is nearly identical to that of full detection. Thus, enabling partial detection of the interfering user results in no noticeable gain from that already achieved by full detection. As shown in Figure 5, the ISB rate regions appear to be close to the OSB rate regions. For ISB, there is a choice of user ordering when performing the maximization in (6) and (11). A different choice of ordering slightly alters the rate regions. Interestingly, the difference between the two orderings decreases when multiuser detection is performed. Figure 7 shows the power allocation for both users for the 


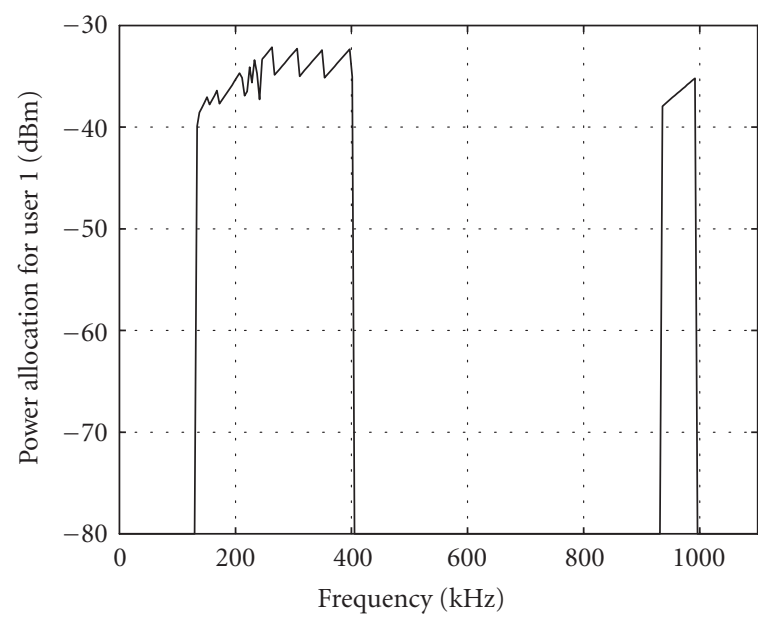

(a)

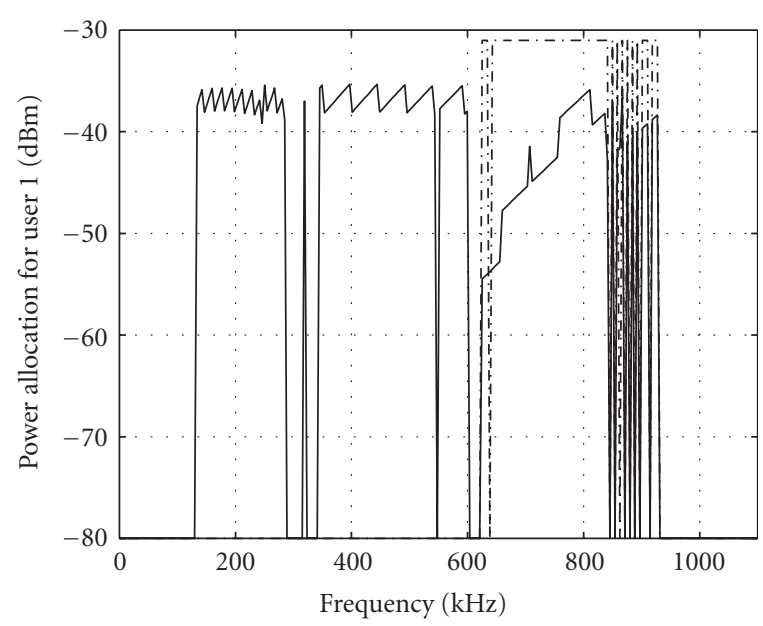

(c)

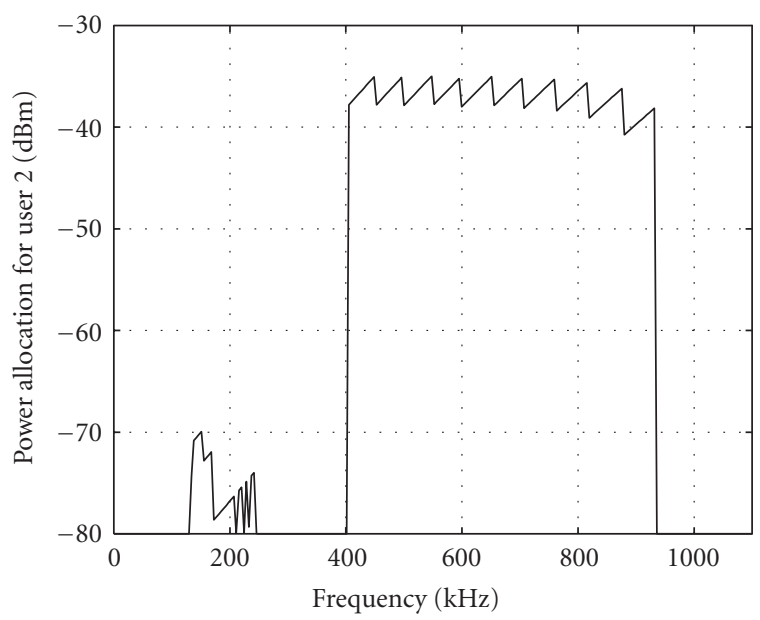

(b)

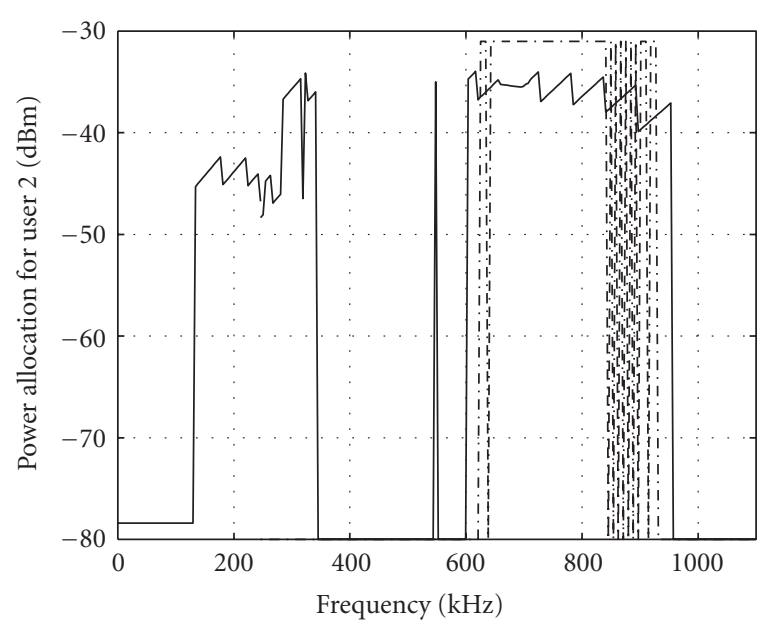

(d)

FIGURE 7: Power allocations for 2-user ADSL downstream (a) and (b) with iterative optimal spectrum balancing alone and (c) and (d) with the joint multiuser detection algorithm (a) and (b) correspond to the rate pair $R_{1}=3.5400 \mathrm{Mbps}, R_{2}=2.8920 \mathrm{Mbps}$; and (c) and (d) correspond to the rate pair $R_{1}=3.4800 \mathrm{Mbps}, R_{2}=3.5400 \mathrm{Mbps}$. The dotted line denotes the frequency band in which multiuser detection is applied.

user $1 \rightarrow 2$ order. Multiuser detection increases the rates from (3.5400 Mbps, 2.8920 Mbps) to (3.4800 Mbps, 3.5400 Mbps) in this case. The power spectra is similar to that resulted from ISB. With more than 2 users, however, the benefit of multiuser detection turns out to be smaller.

Figure 8 illustrates the relationship between the length of the crosstalk channel and the bit rate increase with multiuser detection. The same scenario as depicted in Figure 1 is examined, but with a range of common ADSL line lengths. Both direct channel lengths $l_{1}$ and $l_{2}$ are assumed to be constant in all cases. In general, the performance gain decreases when the ratio $c_{1} / l_{1}$ increases. The maximum gain also increases with the length of the direct channel so that an $8.5 \%$ increase for both users or $17 \%$ increase for one user is possible.

The above simulations are done with an SNR gap of $6 \mathrm{~dB}$ and a margin of $6 \mathrm{~dB}$. Figure 9 shows the performance gain of multiuser detection when the gap and margin is $0 \mathrm{~dB}$. In this case, the benefit of multiuser detection goes as high as $18 \%$ for both users or $36 \%$ for one user. Thus, the benefit of multiuser detection increases when the gap is lowered.

\subsection{VDSL full duplex}

The next set of simulations is for a 2-user VDSL system, as shown in Figure 3, with full duplex transmission. 

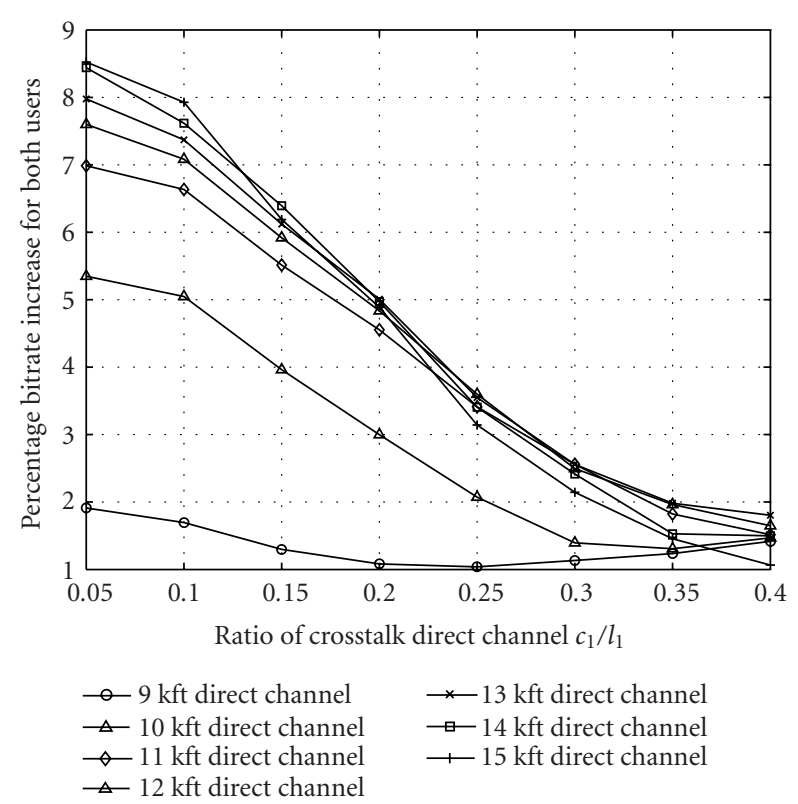

FIgURE 8: Percentage bit rate increase as a function of the direct and crosstalk channel lengths in a 2-user ADSL downstream.

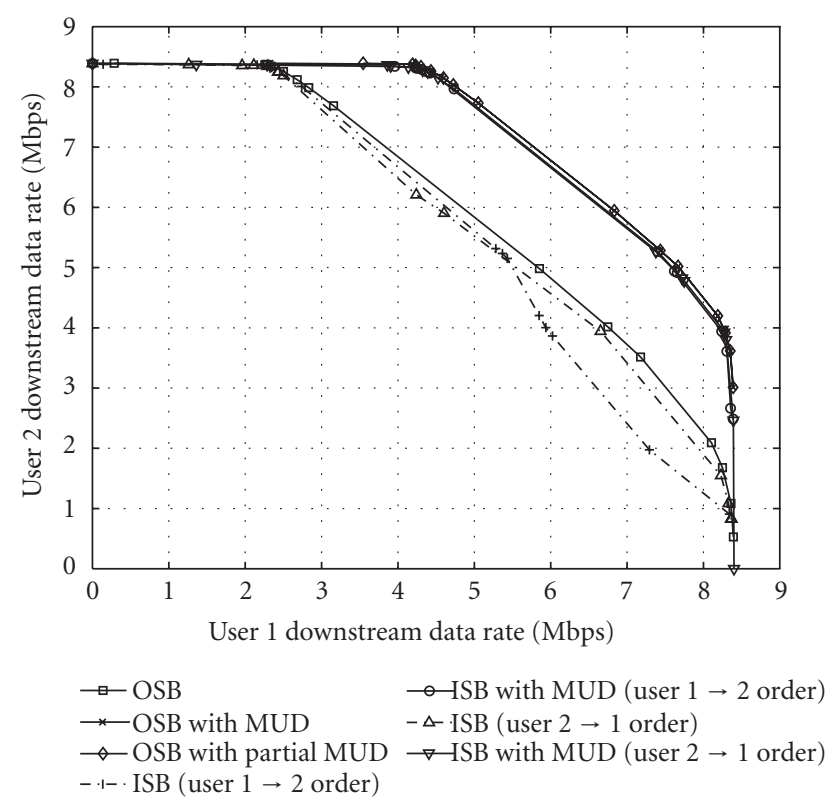

FIgURE 9: Achievable rate region for 2-user ADSL downstream using $\mathrm{OSB} / \mathrm{ISB}$ and the joint multiuser detection algorithm for gap = $0 \mathrm{~dB}$.

Overlapping spectra are allowed between upstream and downstream transmissions. The length of channel two $l_{2}$ is fixed at $2.5 \mathrm{kft}$ while the length of channel one $l_{1}$ varies between 1.5 and $3.7 \mathrm{kft}$. The system is transformed into an equivalent 4-user system as described previously. Only ISB

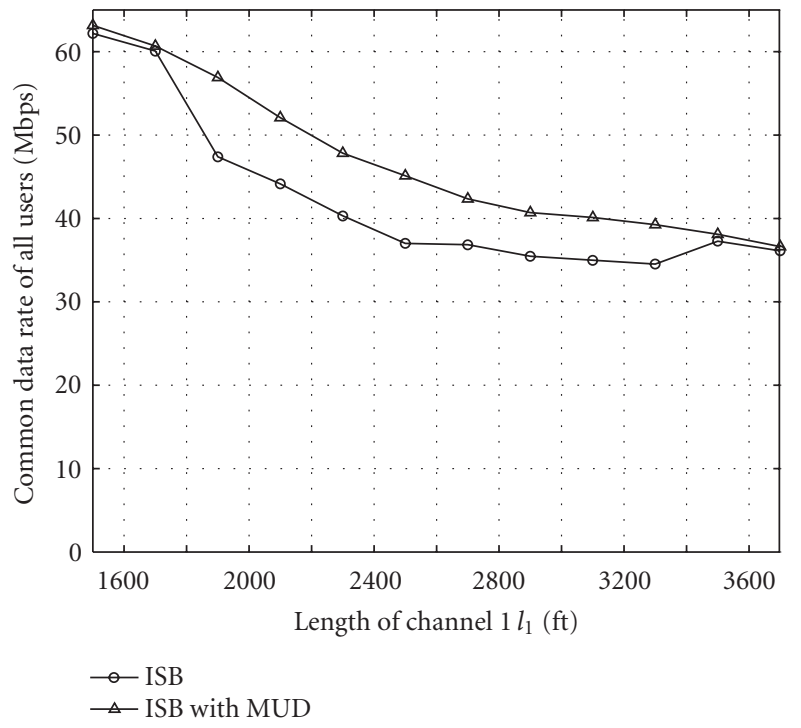

Figure 10: Achievable common bit rate as a function of $l_{1}$ when $l_{2}$ is fixed at $2.5 \mathrm{kft}$ in a 2 -user VDSL full duplex environment. The bit rates of both users in both upstream and downstream directions are kept to be equal.

has been attempted for this scenario because running OSB for a 4-user system is too computationally intensive. Moreover, since the optimization involves the power spectra of an equivalent of four users, the capacity region is fourdimensional, which is difficult to visualize. Alternatively, Figure 10 illustrates the performance gain of multiuser detection when all 4 transmission bit rates are equal. It is found that the performance gain is largest, $22 \%$ for all users, when $l_{1}$ is close to $2.5 \mathrm{kft}$. The reason is that NEXT is strongest when the two channels have equal lengths. In this condition, allowing crosstalk cancellation mitigates the effect of NEXT drastically. Interestingly, the benefit of multiuser detection fades when the difference between $l_{1}$ and $l_{2}$ increases to $1 \mathrm{kft}$.

The power spectrum for each transmission with and without multiuser detection are shown in Figures 11 and 12 respectively. The channel lengths $l_{1}, l_{2}$ are $2.7 \mathrm{kft}$ and $2.5 \mathrm{kft}$. The dotted lines in Figure 11 denote the frequency bands in which multiuser detection is turned on. Without multiuser detection, it is interesting to see that user 1 downstream and user 2 upstream (and similarly user 1 upstream and user 2 downstream) operate in a frequency-division multiplex (FDM) mode. For these two pairs, FDM is optimal because NEXT is too strong for overlapping spectra to occur. With multiuser detection, the cancellation of NEXT becomes a possibility. In this case, overlapping spectra may now be allowed. The extra bits resulting from the overlapping spectra contribute to the performance gain that multiuser detection offers. Note that optimal power spectra are very different from the conventional bandplan where frequencydivision duplex is used to separate upstream and downstream. 


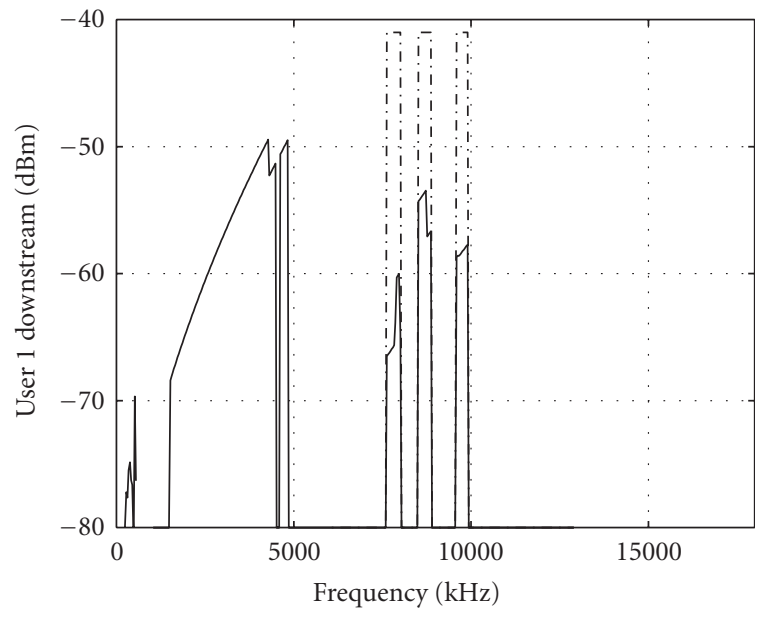

(a)

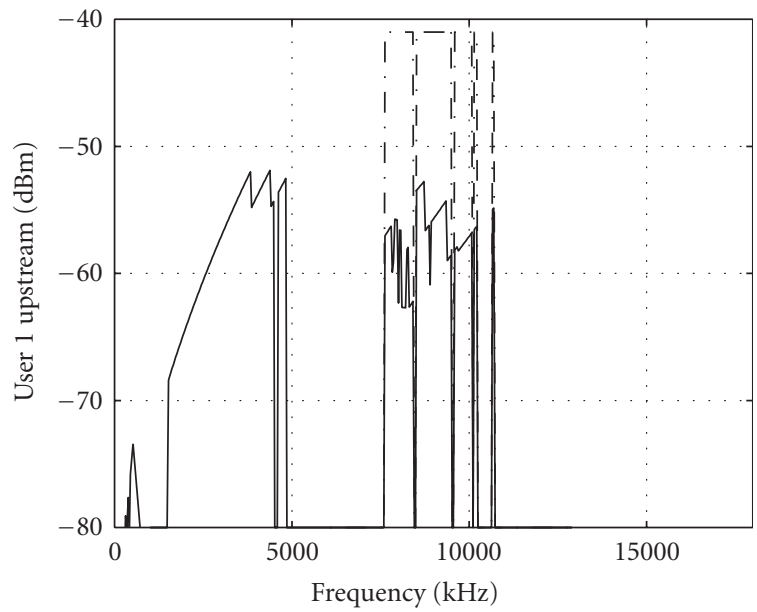

(c)

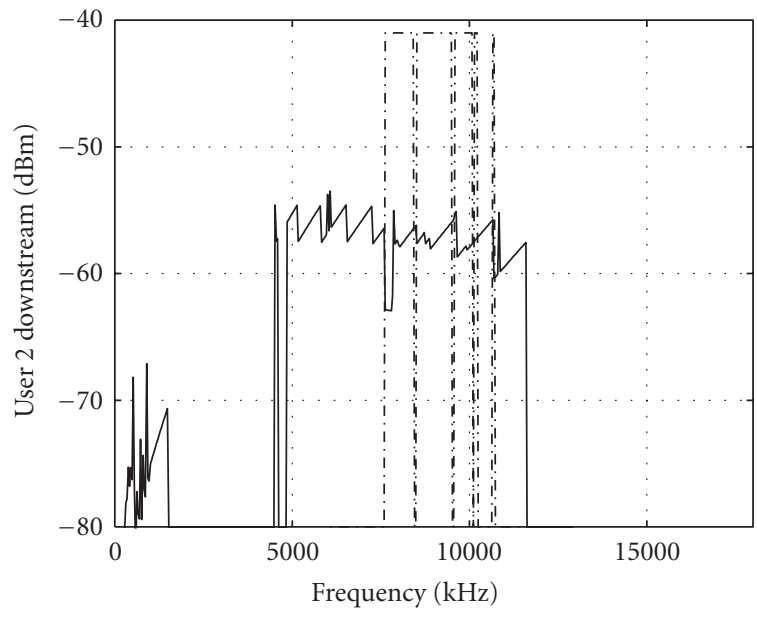

(b)

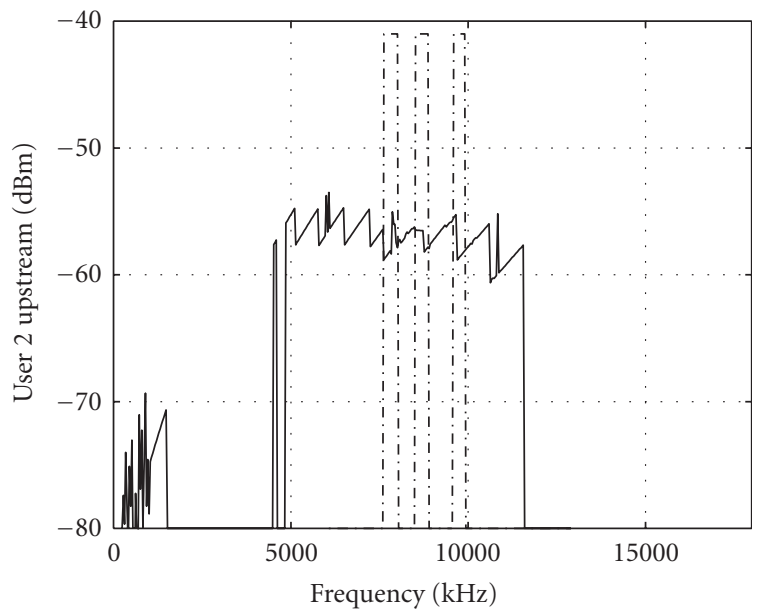

(d)

FIGURE 11: Power allocations for 2-user VDSL downstream with the joint multiuser detection and iterative spectrum balancing algorithm. The channel lengths are set to $l_{1}=2.7 \mathrm{kft}$ and $l_{2}=2.5 \mathrm{kft}$. The four resulting bit rates are equal. The dotted line denotes the frequency band for which the generated NEXT is cancelled by the neighbor user. Full interference detection is assumed.

\section{CONCLUSIONS}

This paper investigates the benefit of multiuser detection and crosstalk cancellation in digital subscriber line systems. Computationally efficient schemes which determine the optimal transmit power spectra and tone selection for multiuser detection are proposed. Multiuser detection is shown to bring a further performance gain than that offered by existing methods. In particular, crosstalk cancellation can be combined with the optimal spectral balancing algorithm for determining the optimal power spectra. Multiuser detection can also be incorporated into the iterative spectral balancing algorithm to deal with complexity concerns when the number of users is large. The possibility of partial detection of the interfering signal has been explored but simulation results show marginal performance gain.

An interesting immediate application of multiuser detection is on VDSL full duplex systems where the interference caused by NEXT is large. Existing systems use frequency-division multiplex to separate the frequency bands for downstream and upstream transmissions. Simulations in this paper suggests that performance gain can be achieved by applying NEXT cancellation to overlapping upstream and downstream bands and at the same time optimally allocating power to minimize the effect of crosstalk. 


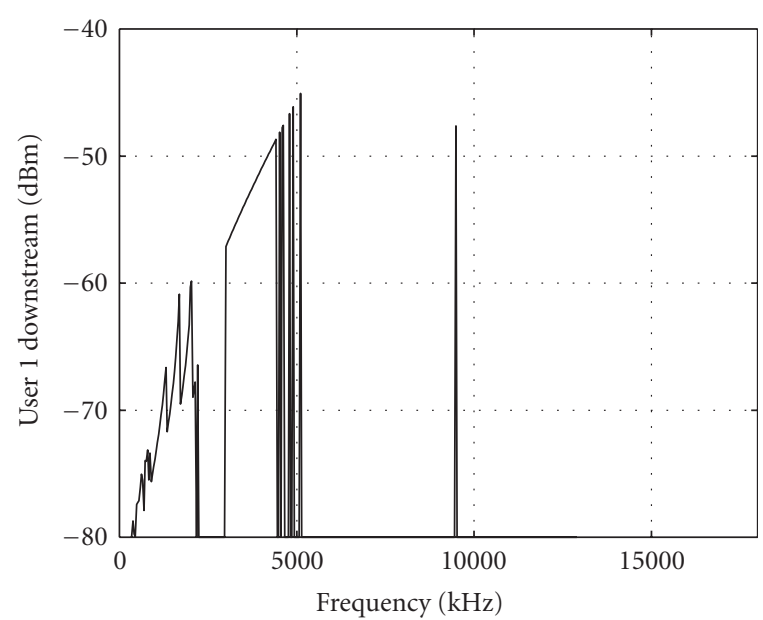

(a)

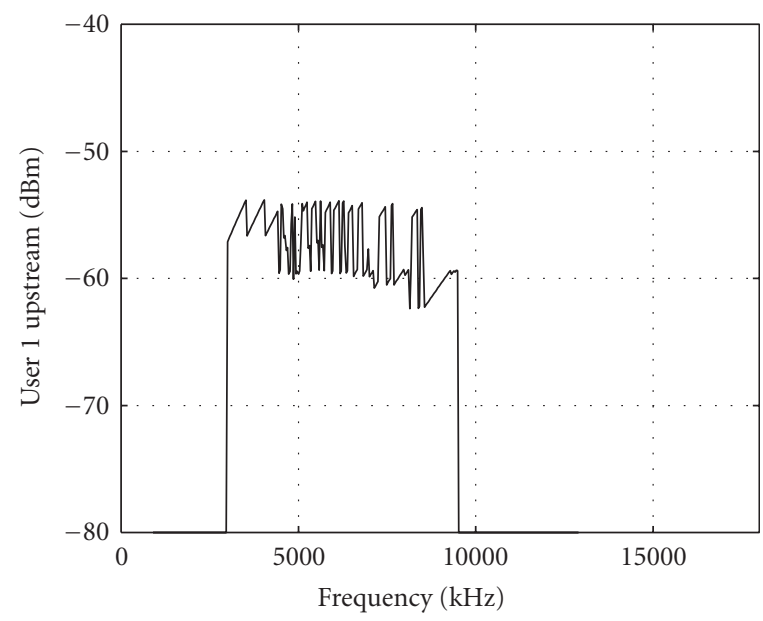

(c)

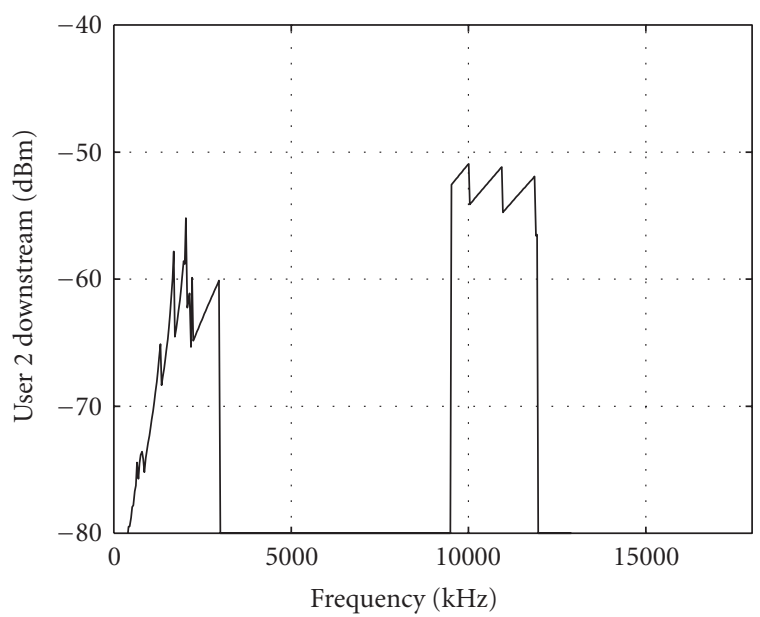

(b)

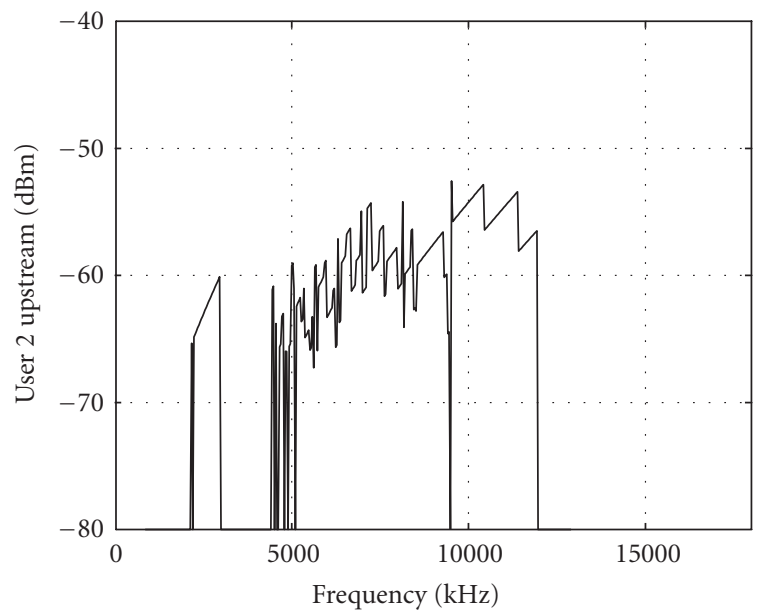

(d)

FIgURE 12: Power allocations for 2-user VDSL full duplex system with iterative optimal spectrum balancing alone. The channel lengths are set to $l_{1}=2.7 \mathrm{kft}$ and $l_{2}=2.5 \mathrm{kft}$, respectively. The four resulting bit rates are equal.

\section{ACKNOWLEDGMENTS}

This work was supported by Bell Canada University Laboratories, Communication and Information Technology Ontario (CITO), and the Natural Science and Engineering Research Council (NSERC) of Canada. This paper has been presented in part at the IEEE International Conference on Acoustics, Speech, and Signal Processing (ICASSP), Philadelphia, 2005.

\section{REFERENCES}

[1] K. B. Song, S. T. Chung, G. Ginis, and J. M. Cioffi, "Dynamic spectrum management for next-generation DSL systems," IEEE Communications Magazine, vol. 40, no. 10, pp. 101-109, 2002.

[2] W. Yu, G. Ginis, and J. M. Cioffi, "Distributed multiuser power control for digital subscriber lines," IEEE Journal on Selected Areas in Communications, vol. 20, no. 5, pp. 1105-1115, 2002.
[3] R. Cendrillon, W. Yu, M. Moonen, J. Verlinden, and T. Bostoen, "Optimal spectrum balancing for digital subscriber lines," to appear in IEEE Trans. Commun., 2006.

[4] G. Ginis and J. M. Cioffi, "Vectored transmission for digital subscriber line systems," IEEE Journal on Selected Areas in Communications, vol. 20, no. 5, pp. 1085-1104, 2002.

[5] W. Yu, R. Lui, and R. Cendrillon, "Dual optimization methods for multiuser orthogonal frequency division multiplex systems," in Proc. IEEE Global Telecommunications Conference (GLOBECOM '04), vol. 1, pp. 225-229, Dallas, Tex, USA, November-December 2004.

[6] R. Lui and W. Yu, "Low-complexity near-optimal spectrum balancing for digital subscriber lines," to appear in IEEE International Conference on Communications (ICC '05), Seoul, Korea, May 2005.

[7] H. Dai and H. V. Poor, "Crosstalk mitigation in DMT VDSL with impulse noise," IEEE Transactions on Circuits and SystemsPart I: Fundamental Theory and Applications, vol. 48, no. 10, pp. 1205-1213, 2001. 
[8] C. Zeng and J. M. Cioffi, "Near-end crosstalk mitigation in ADSL systems," IEEE Journal on Selected Areas in Communications, vol. 20, no. 5, pp. 949-958, 2002.

[9] S. T. Chung and J. M. Cioffi, "The capacity region of frequency-selective Gaussian interference channels under strong interference," in Proc. IEEE International Conference on Communications (ICC '03), vol. 4, pp. 2753-2757, Anchorage, Alaska, USA, May 2003.

[10] T. S. Han and K. Kobayashi, "A new achievable rate region for the interference channel," IEEE Transactions on Information Theory, vol. 27, no. 1, pp. 49-60, 1981.

[11] A. B. Carleial, "Interference channels," IEEE Transactions on Information Theory, vol. 24, no. 1, pp. 60-70, 1978.

[12] F. Sjöberg, M. Isaksson, R. Nilsson, P. Ödling, S. K. Wilson, and P. O. Börjesson, "Zipper: a duplex method for VDSL based on DMT," IEEE Transactions on Communications, vol. 47, no. 8 , pp. 1245-1252, 1999.

[13] R. Nilsson, F. Sjöberg, M. Isaksson, J. M. Cioffi, and S. K. Wilson, "Autonomous synchronization of a DMT-VDSL system in unbundled networks," IEEE Journal on Selected Areas in Communications, vol. 20, no. 5, pp. 1055-1063, 2002.

Vincent M. K. Chan received the B.S. degree in computer engineering from the University of Waterloo, Waterloo, Ontario, Canada, in 2003. He is currently working towards the M.S. degree in electrical engineering at the University of Toronto, Toronto, Ontario, Canada. Through the cooperative education in the University of Waterloo, he worked at Genesis Microchip Inc., Broadcom Corp., and International Busi-

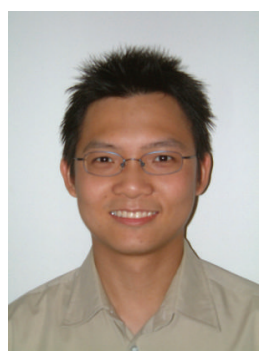
ness Machines (IBM) Corp. His research interests are in the general areas of communication systems and signal processing for digital communications. His current research focuses on multiuser detection and spectrum balancing techniques for digital subscriber lines.

Wei Yu received the B.S. degree in computer engineering and mathematics from the University of Waterloo, Waterloo, Ontario, Canada, in 1997, and the M.S. and Ph.D. degrees in electrical engineering from Stanford University, Stanford, California, USA, in 1998 and 2002, respectively. Since 2002, he has been an Assistant Professor with the Electrical and Computer Engineering Department at the University of

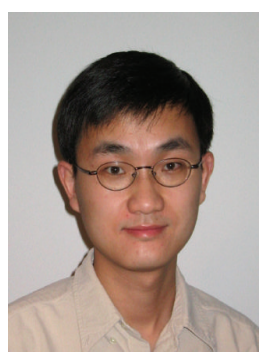
Toronto, Toronto, Ontario, Canada, where he also holds a Canada Research Chair. His main research interests include multiuser information theory, coding, optimization, wireless communications, and broadband access networks. He is currently an Associate Editor for IEEE Transactions on Wireless Communications. 